\title{
Determination of Cnidilin and Its Two Metabolites in Rat Plasma by High-performance Liquid Chromatography-Electrospray Ionization Tandem Mass Spectrometry
}

Authors

Affiliations
Hong Zhu ${ }^{1,2}$, Pengwei Liu ${ }^{1}$, Xiaowei Shi ${ }^{1}$, Huijun $X^{1}{ }^{1}$, Yanping Ren ${ }^{1}$, Qiao Wang ${ }^{1}$, Lantong Zhang ${ }^{1}$

${ }^{1}$ Department of Pharmaceutical Analysis, School of Pharmacy Hebei Medical University, Shijiazhuang, China

${ }^{2}$ Department of Pharmacy, 302 Hospital of People's Liveration Army LA, Beijing, China
Key words

- cnidilin

- HPLC-ESI-MS

- metabolites

- pharmacokinetics

- Radix Angelicae Dahuricae

received July 25,2012

revised October 14, 2012

accepted October 21, 2012

Bibliography

Dol http://dx.doi.org/

10.1055/s-0032-1327983

Published online November 27, 2012

Planta Med 2013; 79: 30-36 (c) Georg Thieme Verlag KC Stuttgart · New York . ISSN 0032-0943

Correspondence

Prof. Dr. Zhang Lantong

Department of Pharmaceutical Analysis

School of Pharmacy

Hebei Medical University

No. 361 Zhongshan Street

Shijiazhuang 050017

China

Phone: + 8631186266419

Fax: + 8631186266419

zhanglantong@263.net

\section{Abstract}

$\nabla$

Simple, fast, and sensitive HPLC coupled with an electrospray ionization tandem mass spectrometry (HPLC-ESI-MS/MS) method for the simultaneous determination of cnidilin and its two metabolites, 3",8-methoxy-isoimperatorin (M1) and 5"-hydroxyl-8-methoxy-isoimperatorin (M2), in rat plasma has been developed. Pimpinellin was used as the internal standard and the total analysis time was $7 \mathrm{~min}$. Methanol was the only reagent during the process of sample handling used as a protein precipitant. The analytes were separated on a reversed-phase C18 column with gradient elution consisting of $0.5 \%$ o aqueous formic

\section{Introduction}

$\nabla$

Radix Angelicae Dahuricae, the dried radix of Angelica dahurica (Fisch. ex Hoffm.) Benth. et Hook. f. and Angelica dahurica (Fisch. ex Hoffm.) Benth. et Hook. f. var. formosana (Boiss.) Shan et Yuan, is a well-known traditional Chinese medicine (TCM). Its dry roots, named baizhi, have been used for the treatment of colds, headaches, toothaches, coryza, psoriasis, and leucorrhea in China for over 2000 years [1]. The main pharmacological activities of Radix Angelicae Dahuricae, antihistamine, photoallergy, promotion of lipometabolism, spasmolysis, antibacterial, inhibition of arachidonic acid-induced platelet aggregation, expansion of coronary vessels, excitation of motor and respiratory center, and anticancer are considered to be related to the major active composition of Radix Angelicae Dahuricae, coumarins [2-4]. The study on coumarin was helpful to understand the pharmacodynamics of Radix Angelicae Dahuricae.

There are many kinds of coumarin in Radix Angelicae Dahuricae, such as oxypeucedanin, bergapten, imperatorin, cnidilin, isoimperatorin, xan- acid and methanol (containing $0.5 \%$ formic acid) in the multiple-reaction monitoring mode. Cnidilin, M1, M2, and the internal standard could be well ionized under positive electrospray ionization conditions. Full validation of the method (linearity, precision, accuracy, limit of detection, and limit of quantification) was carried out and the method was successfully employed in a metabolism study of cnidilin administered to rats at a dose of $24 \mathrm{mg} / \mathrm{kg}$. The pharmacokinetic parameters of cnidilin determined after orally administrating the single coumarin to the rat were obtained and the mass fragmentation pathways for analysis are proposed in this article. 
were found in vivo. M1 and M2 were prepared through a microbial transformation amplification experiment, transformed by Cunninghamella blakesleana AS 3.970. M1 and M2 are both the oxidation products of cnidilin and their chemical structures were identified by ${ }^{1} \mathrm{H}-\mathrm{NMR}$ and ESI-MS $[15,16]$, and named 3",8-methoxy-isoimperatorin and 5"-hydroxyl-8-methoxy-isoimperatorin, repsectively. In this article, an in vivo metabolic study of cnidilin was performed, and the quantification of M1, M2, and cnidilin was achieved on a hybrid quadrupole linear ion trap mass spectrometer in rat plasma after oral administration. The mass fragmentation pathways for the analytes are also proposed in this article. The chemical structures of cnidilin, M1, and M2 are shown in $O$ Fig. 1. The pharmacokinetics of the extraction of Radix Angelicae Dahuricae have been studied many times [17-19]. In this article, the pharmacokinetics parameters of cnidilin determined after orally administrating the single coumarin to a rat were obtained for the first time.

Because of the high sensitivity, short run time, and simple sample pretreatment step, the high-performance liquid chromatography-electrospray ionization tandem mass spectrometry (HPLC-ESI-MS/MS) method has been increasingly used in drug metabolism studies [20-27]. In our experiment, multiple-reaction monitoring (MRM) was employed. In the current study, cnidilin and its metabolites, M1and M2, were successfully quantitatively analyzed in rat plasma.

\section{Materials and Methods}

\section{$\nabla$}

\section{Chemicals and reagents}

The standard sample of cnidilin was isolated by our laborary from Radix Angelicae Dahuricae, and its purity was no less than $98 \%$, as determined by HPLC analysis [8]. M1 and M2 were prepared through a microbial transformation amplification experiment (HPLC purity > 98\%). Pimpinellin [internal standard (IS)] was purchased from the National Institute for the Control of Pharmaceutical and Biological Products (Beijing, China, purity $>98 \%$ ). Methanol alcohol (Fisher) was used for sample pretreatment and the mobile phase, and formic acid (Dima Technology, Inc.) was used for the mobile phase; both were of HPLC grade. Deionized water was produced by the Heal Force-PWVF purification system (Shanghai CanRex Analyse Instrument Co.).

\section{Instrumentation and analytical conditions}

Analysis was performed with an Agilent 1200 series LC system equipped with a quaternary solvent delivery system, an autosampler, and a column compartment. Chromatographic separations were performed with a 5- $\mu \mathrm{m}$ Diamonsil RP-C18 analytical column ( $150 \mathrm{~mm} \times 4.6 \mathrm{~mm})$ and an Alltech C18 precolumn $(4.6 \mathrm{~mm}$ $\times 7.5 \mathrm{~mm} \times 5 \mu \mathrm{m}$ ). The column was operated at room temperature. The mobile phase delivery program of $(\mathrm{A})$ methanol alcohol (containing $0.5 \%$ formic acid) and (B) water (containing $0.5 \%$ formic acid) is as follow: initial $0 \sim 3 \mathrm{~min}$, linear change from $A$ : $B$ $(70: 30, v / v)$ to $A: B(95: 5, v / v) ; 3 \sim 6.5 \mathrm{~min}$, isocratic elution $A: B$ $(95: 5, \mathrm{v} / \mathrm{v}) ; 6.5 \sim 7 \mathrm{~min}$, isocratic elution $\mathrm{A}: \mathrm{B}(70: 30, \mathrm{v} / \mathrm{v})$. This was followed by a 6 -min equilibrium period with initial conditions prior to injection of the next sample. The mobile phase was at a flow rate of $700 \mu \mathrm{L} / \mathrm{min}$. All eluents were filtered through $0.45 \mu \mathrm{m}$ filters before use. $20 \mu \mathrm{L}$ were injected into the instrumentation for analysis.

LC-MS analysis was performed with an Applied Biosystems/MDS Sciex 3200 QTRAP system, a hybrid triple-quadrupole linear ion-

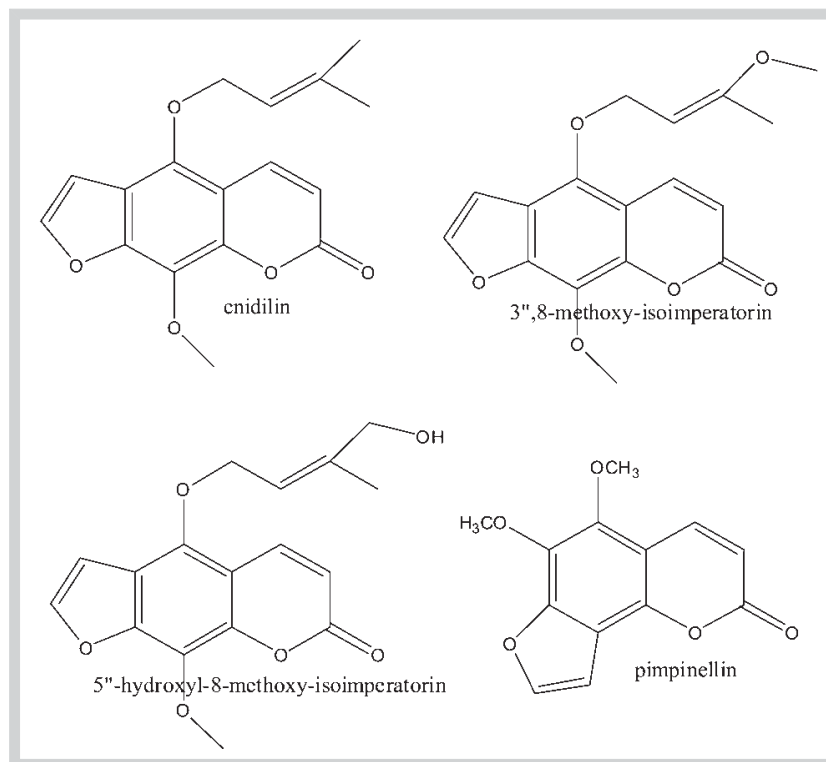

Fig. 1 The chemical structures of cnidilin, 3",8-methoxy-isoimperatorin, 5"'-hydroxyl-8-methoxy-isoimperatorin, and pimpinellin.

trap mass spectrometer equipped with Turbo V sources and Turbolonspray interface. The mass spectrometer was operated using an electrospray ionization source. The ion spray voltage was set to $5500 \mathrm{kV}$ and the turbo spray temperature was maintained at $650{ }^{\circ} \mathrm{C}$. The flow rates of the nebulizer gas (gas 1 ) and the heater gas (gas 2) were set at 60 and 65 arbitrary units, respectively. The curtain gas was kept at 25 arbitrary units and the interface heater was on. Nitrogen was used as a collision gas in all cases. All instrumentations were controlled and analyzed by Analyst software (versions 1.4.2) from Applied Biosystems/MDS Sciex and the multiple-reaction monitoring (MRM) mode was employed for quantification. The entrance potential (EP), ion spray voltage (IS), and cell exit potential (CXP) for analysis were $10 \mathrm{~V}, 5500 \mathrm{~V}$, $3 \mathrm{~V}$, respectively. And the precursor-to-product ion pairs, declustering potential (DP), and collision energy (CE) were 301.2/233.1, $46 \mathrm{~V}, 16 \mathrm{eV}$ for cnidilin, 317.2/231.1, 20 V, $19 \mathrm{eV}$ for M1, 317.1/ 233.2, $20 \mathrm{~V}, 12 \mathrm{eV}$ for M2, and 247.1/231.1, $20 \mathrm{~V}, 19 \mathrm{eV}$ for IS, respectively.

\section{Preparation of standard solution and quality control samples}

Cnidilin, M1, and M2 were separately weighed to the appropriate amount and dissolved in methanol to make the stock solutions. Then, the three stock solutions were mixed and diluted with methanol to prepare a final mixed standard solution containing $6.95 \mu \mathrm{g} / \mathrm{mL}$ of cnidilin and $1.25 \mu \mathrm{g} / \mathrm{mL}$ of M1 and M2. The final mixture was diluted with methanol to make a series of working solutions at appropriate ratios to yield concentrations which were used as calibration standard samples. Pimpinellin was diluted with methanol to obtain an IS solution at the concentration of $2.07 \mu \mathrm{g} / \mathrm{mL}$. Quality control (QC) samples containing low, medium, and high concentrations of cnidilin, M1, and M2 were prepared with methanol. 


\section{Pretreatment of plasma sample}

Venous blood from healthy, adult, male Sprague-Dawley rats (Certificate No. DK 0908083, about $250 \mathrm{~g}$ body weight), provided by the Experimental Animals Center of Hebei Province (Shijiazhuang, China), was collected from the eye canthus. Before dosing, all of the experimental animals were fasted for $12 \mathrm{~h}$. Suspension of cnidilin, prepared by $0.5 \%$ CMC-Na (sodium carboxymethylcellulose), was $3 \mathrm{mg} / \mathrm{mL}$ dose. Then the prepared suspensions were orally administrated to 6 rats at a dose of $24 \mathrm{mg} / \mathrm{kg}$. Blood samples $(0.2 \mathrm{~mL})$ were obtained from each rat $15,30,60,120,180$, $240,300,360,420,480$ and $600 \mathrm{~min}$ after administration and collected in heparinized centrifuge tubes. Then the blood samples were centrifuged at $12000 \mathrm{rpm}$ for $10 \mathrm{~min}$ and the plasma was frozen at $-20^{\circ} \mathrm{C}$ until analysis. A pool of plasma from one rat orally administrated $0.5 \%$ CMC-Na was used as blank plasma. The protocols of animal experiments were approved by the Animal Center of Hebei Medical University. The animal application approval number was 0000302, which was given by the Medical Ethics Committee of Hebei Medical University in May 2011.

$160 \mu \mathrm{L}$ methanol, used as a protein precipitant, was added to $50 \mu \mathrm{L}$ of the plasma sample. After the addition of $20 \mu \mathrm{L}$ IS and $20 \mu \mathrm{L}$ blank methanol, the tubes were vortexed for $30 \mathrm{~s}$, and then centrifuged at $12000 \mathrm{rpm}$ for $10 \mathrm{~min} .20 \mu \mathrm{L}$ of the supernatant were saved for analysis.

\section{Method validation}

Calibration standard plasma samples and QC plasma samples were prepared as described above, except that $50 \mu \mathrm{L}$ blank plasma was used instead of plasma samples, $20 \mu \mathrm{L}$ calibration standard samples and $20 \mu \mathrm{L}$ QC samples were used instead of blank methanol. The calibration curves were constructed for seven concentrations in triplicate. Calibrations curves were constructed using the analyte and IS peak-area ratios via a weighted $\left(1 / \mathrm{x}^{2}\right)$ leastsquares linear regression in the form of $y=a x+b$. Unknown sample peak-area ratios were then interpolated from the calibration curve to provide the concentrations of analytes.

The lower limit of detection (LLOD) was defined at a signal-tonoise $(\mathrm{S} / \mathrm{N})$ ratio of $3: 1$ and the lower limit of quantification (LLOQ) was defined as the lowest quantifiable concentration of the analyte.

The intraday and inter-day precision were evaluated by replicative analysis of five sets of samples spiked with QC plasma samples at three concentration levels of cnidilin, M1, and M2 within a day or during three consecutive days, and the precision was denoted by the relative standard deviation (R.S.D.). The accuracy was determined by the three levels of QC plasma samples in five replicates, and the accuracy was expressed by relative error (R.E.).

In the method stability test, the stability of cnidilin, M1, M2, and IS stock solutions was evaluated after storage at room temperature for $24 \mathrm{~h}$. In the biological samples stability test, short-term stability was assessed by analyzing QC plasma samples kept at room temperature for $24 \mathrm{~h}$ that exceeded the routine preparation time of samples, long-term stability was determined by assaying QC plasma samples after storage at $-20^{\circ} \mathrm{C}$ for 30 days, and freeze-thaw stability was investigated after three freeze-thaw cycles, which consisted of storage at $-20^{\circ} \mathrm{C}$ for a minimum of $24 \mathrm{~h}$ followed by thawing at room temperature. Each analyte was determined at least three times.

The extraction recovery of analytes was determined by replicative analysis of five sets by comparing the blank plasma spiked with the QC samples at three concentrations after extraction with the blank plasma added to the same sample before extraction. The quantification of the chromatogram was performed by using the peak area of the analytes. The determination of the extraction recovery of IS was similar to that of the analytes.

The matrix effect was evaluated for detecting the influence of matrix compounds to analytes $[28,29]$. The matrix effect can be expressed by $\mathrm{A} / \mathrm{B} \times 100$. A was the blank plasma spiked with $\mathrm{QC}$ samples at three concentrations, and B was the QC samples at equivalent concentrations. Three different concentration levels of analytes were evaluated by analyzing five times.

\section{Results and Discussion \\ $\nabla$}

In this study, it was found that cnidilin, M1, M2, and IS could be well ionized under positive electrospray ionization conditions. The mass spectra of the analytes are shown in 0 Fig. 2. Considering the intensity, $\mathrm{m} / \mathrm{z} 301.2 \rightarrow 233.1$ for cnidilin, $\mathrm{m} / \mathrm{z} 317.2 \rightarrow$ 231.1 for M1, $m / z 317.1 \rightarrow 233.2$ for M2, and $m / z 247.1 \rightarrow 231.1$ for IS were chosen for quantification. The product ion scan spectra of cnidilin, M1, M2, and IS are shown in $\odot$ Fig. 2.

Over the concentration range of $25 \mathrm{ng} / \mathrm{mL} 3200 \mathrm{ng} / \mathrm{mL}$ for cnidilin and $3.91 \mathrm{ng} / \mathrm{mL} 500 \mathrm{ng} / \mathrm{mL}$ for M1 and M2 in rat plasma, the standard curves showed good linearity. The regression equation of calibration curves and their correlation coefficients $\left(r^{2}\right)$ were $\mathrm{y}=0.7956 \mathrm{x}-0.0007$ and 0.9991 for cnidilin, $\mathrm{y}=0.0433 \mathrm{x}+$ 0.0003 and 0.9975 for M1, and $y=0.3132 x+0.0004$ and 0.9997 for $\mathrm{M} 2$, respectively. The relative error from a $1 / \mathrm{x}^{2}$ weighted least-square linear regression line varied from $-11.8 \%$ to $13.5 \%$ for each point of the seven calibration curves of cnidilin, from $-12.7 \%$ to $13.7 \%$ for that of M1, and from $10.1 \%$ to $11.5 \%$ for that of M2. The LLOD was $0.156 \mathrm{ng} / \mathrm{mL}$ for cnidilin, and $0.195 \mathrm{ng} / \mathrm{mL}$ for M1 and M2, observed under the optimized conditions.

The intraday precision was evaluated by the three levels of QC plasma samples on three sequential runs in five replicates and ranged between $0.9 \%$ and $2.7 \%, 1.1 \%$ and $3.2 \%$, and $1.2 \%$ and $1.9 \%$ for cnidilin, M1, and M2, respectively. The inter-day precision was obtained in three consecutive days and varied from $1.3 \%$ to $2.2 \%, 2.9 \%$ to $4.1 \%, 0.6 \%$ to $2.6 \%$ for cnidilin, M1, and M2, respectively. The accuracy was evaluated by the three levels of QC plasma samples in five replicates and the R.E. of all the QC plasma samples was less than 5.8\%. Details are shown in O Table 1.

The extraction recovery of three analytes at low, medium, and high concentrations was in the range of $98.7 \%$ 103.6\% with an R.S.D. value of $3.6 \%$ for cnidilin, $97.3 \%$ 99.3\% with an R.S.D. value of $4.1 \%$ for M1, and $95.5 \% ~ 101.9 \%$ with an R.S.D. value of $2.8 \%$ for M2 $(\mathrm{n}=5)$. The results, as shown in 0 Table 1 , indicated that the three analytes could be completely extracted and met the requirements of the extraction recovery test.

The data of the matrix effect test is also shown in $\odot$ Table 1 . As can be seen from this data, the ME\% were all less than $15 \%$, which is acceptable.

The results of short-term, long-term, and freeze-thaw cycle stability of cnidilin, M1, and M2 in plasma are summarized in 0 Table 2. Through the data, we can see that cnidilin, M1, and M2 were stable in plasma because, at any QC level, the results well met the criterion for the stability test.

The selectivity of the method was evaluated by comparing blank plasma samples obtained from six rats with the spiked plasma. - Fig. 3 shows the typical chromatograms of a blank plasma, a blank plasma sample spiked with the QC sample and the IS, and 


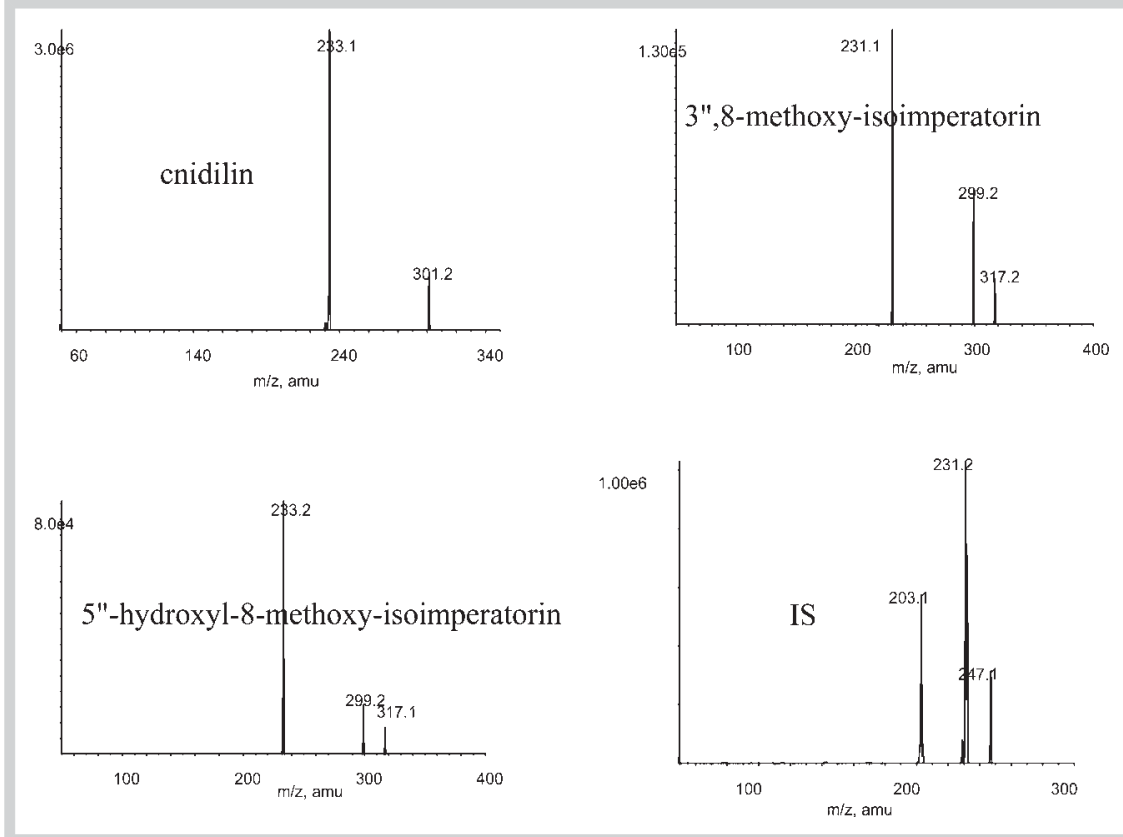

Fig. 2 The product ion scan spectra of cnidilin, 3",8-methoxy-isoimperatorin, 5"'-hydroxyl-8methoxy-isoimperatorin, and pimpinellin in the positive mode.

Table 1 The precision, accuracy, ME, and extraction recovery of analytes $(n=5)$.

\begin{tabular}{|c|c|c|c|c|c|c|}
\hline \multirow[t]{3}{*}{ Components } & \multirow[t]{3}{*}{ The level of QC } & \multicolumn{2}{|c|}{ Precision (RSD \%) } & \multirow{3}{*}{$\begin{array}{l}\text { Accuracy } \\
\text { (RE \%) }\end{array}$} & \multirow{3}{*}{$\begin{array}{l}\text { Extraction } \\
\text { recovery (\%) }\end{array}$} & \multirow[t]{3}{*}{ ME (\%) } \\
\hline & & & & & & \\
\hline & & Intraday & Inter-day & & & \\
\hline \multirow[t]{3}{*}{ Cnidilin } & Low & 2.7 & 2.2 & -2.1 & 100.4 & 101.4 \\
\hline & Medium & 1.8 & 1.7 & 3.7 & 98.3 & 98.7 \\
\hline & High & 0.9 & 1.3 & 4.1 & 99.3 & 103.6 \\
\hline \multirow[t]{3}{*}{ 3",8-methoxy-isoimperatorin } & Low & 3.2 & 3.6 & 5.8 & 103.2 & 99.3 \\
\hline & Medium & 1.1 & 2.9 & -1.5 & 101.1 & 98.7 \\
\hline & High & 2.5 & 4.1 & 1.9 & 97.4 & 97.3 \\
\hline \multirow[t]{3}{*}{ 5"-hydroxyl-8-methoxy-isoimperatorin } & Low & 1.4 & 2.6 & 2.6 & 98.7 & 95.5 \\
\hline & Medium & 1.2 & 1.0 & 4.8 & 99.5 & 99.1 \\
\hline & High & 1.9 & 0.6 & -3.2 & 99.7 & 101.9 \\
\hline
\end{tabular}

Table 2 The stability of the analytes $(n=5)$.

\begin{tabular}{|c|c|c|c|c|c|c|c|}
\hline \multirow[t]{3}{*}{ Components } & \multirow[t]{3}{*}{ The level of QC } & \multicolumn{6}{|c|}{ Biological samples stability } \\
\hline & & \multicolumn{2}{|c|}{ Short-term } & \multicolumn{2}{|l|}{ Long-term } & \multicolumn{2}{|l|}{ Freeze-thaw } \\
\hline & & $\begin{array}{l}\text { Mean } \pm \text { SD } \\
(\mathrm{ng} / \mathrm{mL})\end{array}$ & $\begin{array}{l}\text { RE } \\
\text { (\%) }\end{array}$ & $\begin{array}{l}\text { Mean } \pm \text { SD } \\
(\mathrm{ng} / \mathrm{mL})\end{array}$ & $\begin{array}{l}\text { RE } \\
\text { (\%) }\end{array}$ & $\begin{array}{l}\text { Mean } \pm \text { SD } \\
(\mathrm{ng} / \mathrm{mL})\end{array}$ & $\begin{array}{l}\text { RE } \\
\text { (\%) }\end{array}$ \\
\hline \multirow[t]{3}{*}{ Cnidilin } & Low & $50.41 \pm 0.05$ & 2.4 & $49.78 \pm 0.04$ & -3.6 & $50.17 \pm 0.03$ & -4.1 \\
\hline & Medium & $398.17 \pm 0.02$ & 4.0 & $400.98 \pm 0.02$ & 1.3 & $399.10 \pm 0.05$ & -1.5 \\
\hline & High & $2562.51 \pm 0.04$ & -1.2 & $2562.84 \pm 0.02$ & -4.2 & $2558.95 \pm 0.01$ & 4.7 \\
\hline \multirow{3}{*}{$\begin{array}{l}3{ }^{\prime \prime}, 8 \text {-methoxy-isoimper- } \\
\text { atorin }\end{array}$} & Low & $7.90 \pm 0.07$ & 3.8 & $7.80 \pm 0.01$ & 3.6 & $7.83 \pm 0.04$ & 3.7 \\
\hline & Medium & $62.94 \pm 0.06$ & -2.9 & $62.43 \pm 0.04$ & 0.5 & $62.59 \pm 0.02$ & 2.8 \\
\hline & High & $400.80 \pm 0.05$ & -3.1 & $399.57 \pm 0.02$ & 2.6 & $400.10 \pm 0.05$ & -2.0 \\
\hline \multirow{3}{*}{$\begin{array}{l}\text { 5"-hydroxyl-8-methoxy- } \\
\text { isoimperatorin }\end{array}$} & Low & $7.83 \pm 0.03$ & 4.8 & $7.81 \pm 0.04$ & -3.9 & $7.82 \pm 0.03$ & 1.1 \\
\hline & Medium & $62.15 \pm 0.04$ & -2.6 & $62.55 \pm 0.03$ & -4.4 & $62.47 \pm 0.01$ & -3.4 \\
\hline & High & $399.00 \pm 0.10$ & 1.9 & $400.21 \pm 0.00$ & -3.2 & $399.85 \pm 0.03$ & 4.1 \\
\hline
\end{tabular}

a test plasma sample. All samples were found to be free of interferences with the analytes and IS.

Because of the low plasma concentration of metabolites, solidphase extraction (SPE) and liquid-liquid extraction (LLE) were often used in plasma sample preparation $[20,24,26]$. But in our study, methanol was the only additive in the process of sample treatment, which used as a protein precipitant. In this way, not only the signal intensity could meet the testing requirements, but also the sample preparation process was simple. Additionally, 

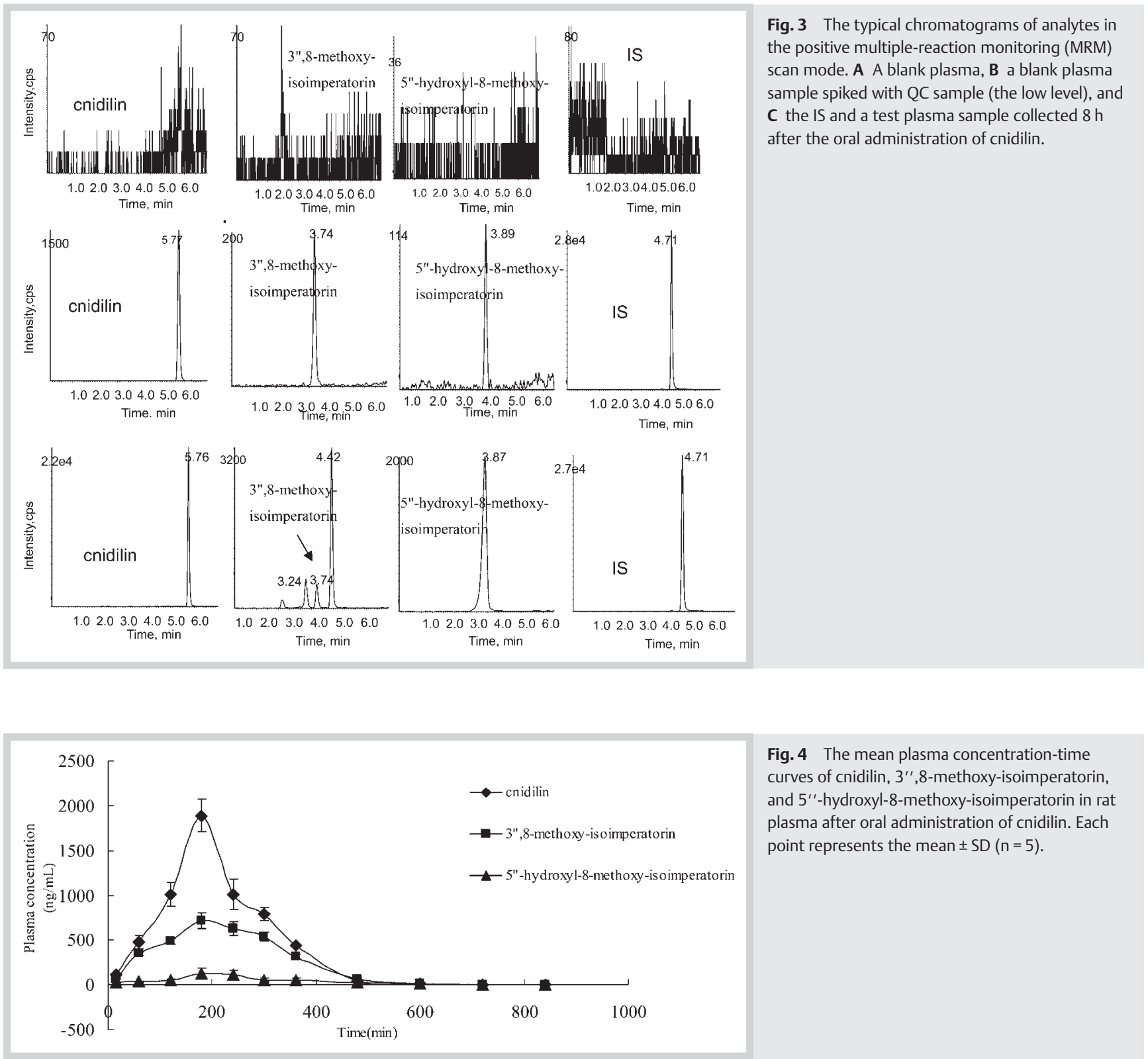

Fig. 4 The mean plasma concentration-time curves of cnidilin, 3",8-methoxy-isoimperatorin, and $5^{\prime \prime}$-hydroxyl-8-methoxy-isoimperatorin in rat plasma after oral administration of cnidilin. Each point represents the mean \pm SD $(n=5)$.

fewer additives meant less interference. There was no solvent peak in the chromatogram and good recoveries were obtained. Pimpinellin, which also belongs to coumarins, was chosen as the internal standard. And its chemical structure and chromatographic behavior were similar to that of the analytes. The chemical structure of pimpinellin is shown in $\bullet$ Fig. 1.

To obtain the optimal chromatographic conditions, HPLC parameters including category of column (Diamonsil C18, $250 \mathrm{~mm} \times$ $4.6 \mathrm{~mm}, 5 \mu \mathrm{m}$, SunFire C18, $150 \mathrm{~mm} \times 4.6 \mathrm{~mm}, 5 \mu \mathrm{m}$ or Diamonsil C18, $150 \mathrm{~mm} \times 4.6 \mathrm{~mm}, 5 \mu \mathrm{m}$ ), mobile phase (methanol-water, acetonitrile-water, methanol- $0.1 \%$ formic acid aqueous solution, acetonitrile- $0.1 \%$ formic acid aqueous solution, methanol containing $0.05 \%$ formic acid- $0.05 \%$ formic acid aqueous solution or acetonitrile containing $0.05 \%$ formic acid- $0.05 \%$ formic acid aqueous solution), and the flow rate of the mobile phase $(0.5$, $0.6,0.7,0.8 \mathrm{~mL} / \mathrm{min}$ ) were all examined and compared. Lastly, the Diamonsil C18 column $(150 \mathrm{~mm} \times 4.6 \mathrm{~mm}, 5 \mu \mathrm{m})$ and the flow rate of $0.7 \mathrm{~mL} / \mathrm{min}$ were chosen to be the optimal chromatographic conditions, which could provide higher separation. Be- cause of gradient elution, formic acid added in both the aqueous phase and the organic phase could keep the ratio of acid in the mobile phase in the process of analysis, which is why methanol containing $0.05 \%$ formic acid- $0.05 \%$ formic acid aqueous solution was chosen as the optimal mobile phase.

In this study, we successfully determined the cnidilin, M1, and M2 in rat plasma after administrating cnidilin to rats at a dose of $24 \mathrm{mg} / \mathrm{kg}$. The plasma concentration-time curves of cnidilin, M1, and M2 are shown in Fig. 4. As can be seen in the figure, the variation of plasma concentration of metabolites was equal to the parent compound cnidilin, but much lower than the plasma concentration of cnidilin. This indicates that cnidilin may be degraded or biotransformed into another metabolite. M1 and M2 are both oxidative metabolites of cnidilin. The other metabolic forms of cnidilin need further research.

The pharmacokinetic parameters of cnidilin are shown in $\triangle$ Table 3. The $k e$ is the slope of the last four points from the plasma concentration-time curve. The $t_{1 / 2}$ was obtained by $0.693 / k e$. $\mathrm{AUC}_{0-t}$ was calculated by the Trapezoidal Rule. $\mathrm{AUC}_{0-\infty}$ was calculated by 
Table 3 The pharmacokinetic parameters of cnidilin, 3",8-methoxy-isoimperatorin, and 5"-hydroxyl-8-methoxy-isoimperatorin.

\begin{tabular}{|llcc}
\hline Pharmacokinetic parameters & Mean \pm SD & & \\
& Cnidilin & $\mathbf{3}^{\prime \prime}, \mathbf{8}$-methoxy-isoimperatorin & 5"-hydroxyl-8-methoxy-isoimperatorin $^{\prime \prime}$ \\
$C_{\max }(\mathrm{ng} / \mathrm{mL})$ & $1890.0 \pm 0.5$ & $715.0 \pm 0.6$ & $126.0 \pm 0.8$ \\
\hline$T_{\max }(\mathrm{h})$ & $3.0 \pm 0.0$ & $3.0 \pm 0.0$ & $3.0 \pm 0.0$ \\
\hline$k e\left(\mathrm{~h}^{-1}\right)$ & $0.1192 \pm 0.0170$ & $0.1145 \pm 0.0057$ & $0.1183 \pm 0.0070$ \\
\hline$t_{1 / 2}(\mathrm{~h})$ & $5.81 \pm 0.75$ & $6.05 \pm 0.46$ & $5.86 \pm 0.60$ \\
\hline $\mathrm{AUC}_{0-\mathrm{t}}(\mathrm{ng} \mathrm{h} / \mathrm{mL})$ & $8446.4 \pm 0.8$ & $3361.4 \pm 0.2$ & $556.1 \pm 0.1$ \\
\hline $\mathrm{AUC}_{0-\infty}(\mathrm{ng} \mathrm{h} / \mathrm{mL})$ & $8454.8 \pm 0.4$ & $3370.1 \pm 0.1$ & $564.6 \pm 0.1$ \\
\hline
\end{tabular}

$\mathrm{AUC}_{0-t}+\mathrm{Cn} / \mathrm{ke}$. The differences of pharmacokinetics between the single coumarins and the extraction of Radix Angelicae Dahuricae are still being studied. Comparing the pharmacokinetic parameters with imperatorin [19], we found that they have the same $T_{\max }$, but the elimination rate of cnidilin was faster than that of imperatorin. On the contrary, $t_{1 / 2}$ of cnidilin was slower than that of imperatorin.

In this study, a method for simultaneous determination of cnidilin and its two metabolites in rat plasma has been validated for the first time. With this method the total analysis time was 7 min, which made it possible to analyze a large number of biological samples at one time. The pretreatment method of the plasma sample was simple and only the addition of methanol was used as a protein precipitant. The addition of formic acid in the organic phase could maintain the ratio of the mobile phase during the whole analysis process. M1 and M2 were found in rat plasma for the first time, and could be reliably assayed with a coupled HPLC-ESI-MS/MS system. In this way, there was no signal interference from endogenous compounds. This is the first time that the in vivo metabolism has been determined and the pharmacokinetic parameters obtained after orally administrating the single active ingredient extracted from the TCM. This simple, sensitive, and stabile method described above has been successfully applied to the metabolism research of cnidilin, and this is the first time coumarin and its metabolites have been determined in rat plasma with the LC-MS method, which could be helpful in studying the metabolism of coumarins or the metabolism of Radix Angelicae Dahuricae.

\section{Acknowledgements}

$\nabla$

The work was financially supported by the National Natural Science Foundation of China (81102412), the Ministry of Education Key Project of Science and Technology Foundation of China (211021) and the Natural Science Foundation of Hebei Province - CSPC Pharmaceutical Joint Research Fund of China (H2012206079).

\section{Conflict of Interest \\ $\nabla$}

All the authors have declared no conflict of interest.

\section{References}

1 The Pharmacopoeia Commission of PRC. The pharmacopoeia of the People's Republic of China. Part I. Beijing: Chinese Medicine Science and Technology Press; 2010: 97

2 Kang K, Kong C, Seo Y, Kim M, Kim S. Anti-inflammatory effect of coumarins isolated from Corydalis heterocarpa in HT-29 human colon carcinoma cells. Food Chem Toxicol 2009; 47: 2129-2134
3 Hao G, Wang Z, Fu W, Yang Y. Research progress on effect of coumarins compounds in anti-tumor. China J Chin Mater Med 2008; 33: 20162019

4 Du XX, Wang CM, Qin J, Duan DH. Progress of study on coumarin of Angelicae dahuricae. J Jinggangshan Univ (Natural Sciences) 2005; 26: $120-122$

5 Zhao XZ, Jia XD, Chen J, Wang M, Dong YF, Feng X. Studies on chemical constituents of Angelica dahurica. Lishizhen Med Mater Med Res 2008; 19: 2000-2002

6 Yang J, Deng Y, Zhou Z, Wu F. Chemical constituents of Angelica dahurica. Chem Res Appl 2002; 14: 227-229

7 Janine Z, Eva E, Diana C, Steffen H, Matthias H. HPLC-based activity profiling of Angelica pubescens roots for new positive $\mathrm{GABA}_{\mathrm{A}}$ receptor modulators in Xenopus oocytes. Fitoterapia 2011; 82: 434-440

8 Zheng XG, Zhang XW, Sheng XN, Yuan ZF, Yang W, Wang $Q$, Zhang LT. Simultaneous characterization and quantitation of 11 coumarins in Radix Angelicae Dahuricae by high performance liquid chromatography with electrospray tandem mass spectrometry. J Pharm Biomed Anal 2010; 51: 599-605

9 Franke K, Porzel A, Masaoud M, Adam G. Furanocoumarins from Dorstenia gigas. Phytochemistry 2001; 56: 611-621

10 Yang $W$, Ye M, Liu M, Kong DZ, Shi R, Shi XW, Zhang KR, Wang $Q$ Z Zhang $L T$. A practical strategy for the characterization of coumarins in Radix Glehniae by liquid chromatography coupled with triple quadrupolelinear ion trap mass spectrometry. J Chromatogr A 2010; 1217: 45874600

11 Zhong F, Wang WM, Chen N. Metabolomics and its application in vivo studies of Chinese medicine. Chin J Integr Tradit Western Nephrol 2008; 9: 920-922

12 Chen JZ. Progress in pharmacokinetics of Chinese medicine. J Strait Pharm 2007; 19: 12-14

13 Ming $Q$. Overview of metabolism of Chinese medicine. J Jiujiang Med 2007; 22: 48-52

14 Chai ZW, Pan GX. A brief review on the method of medical metabolism. Tianjin J Tradit Chin Med 2006; 23: 83-85

15 Zhu H, Zhang LT, Ren YP, Xu HJ, Cui Y. Microbial transformation of cnidilin and identification of its metabolites. Chin J Pharm 2011; 42: 112115

16 Kong DZ, Han XJ, Jing XJ, Wang N, Zhang LT. Study on metabolites of mnisoldipine by microbial transformation. Chin J Pharm Anal 2010; 30 : 204-207

17 Huang YW, Chen XH, Huo YS, Bi KS. Pharmacokinetic study of imperatorin in rat after oral administration of Extractum Angelicae Dahuricae Siccum. J Shenyang Pharm Univ 2008; 25: 215-218

18 Rong H, Li ZL. Phamacokinetic study of isoimperatorin in rats after drug administration. Chin J Inf TCM 2009; 16: 40-41

$19 \mathrm{Li} \mathrm{YB}$. Determination of imperatorin in rat plasma by reversed-phase high-performance liquid chromatography after oral administration of Radix Angelicae dahuricae extract. J Pharm Biomed Anal 2006; 40: 1253-1256

20 Kong DZ, Li SN, Zhang XW, Gu JM, Liu M, Meng Y, Fu Y, La XJ, Xue GQ Zhang $L T$, Wang $Q$. Simultaneous determination of $m$-nisoldipine and its three metabolites in rat plasma by liquid chromatography-mass spectrometry. J Chromatogr B 2010; 878: 2989-2996

$21 \mathrm{Fu}$ NG, Chen F, Wei N, Ren SZ, Liu MS, Zhang JQ. LC-MS determination of brief quercetin metabolites in rat plasma. Chin J Pharm Anal 2009; 29: 764-768

22 Pang LQ Liang QL, Liu QF, Ran XR, Wang YM, Luo GA. Identification of metabolites of complicated Qingkailing in rat. Chin J Anal Chem 2007; 35: $1421-1424$ 
23 Chen F, Fu NG, Ren SZ, Wei N, Zhang JQ. Determination of quercetin metabolites in rat plasma by liquid chromatography-mass spectrometry. J Chin Pharm 2008; 43: 225-227

24 Cao LY, Li YS, Chen ZJ, Li YK. The metabolic study of glaucocalyxin A in rat in vivo and in vitro. West China J Pharm Sci 2006; 21: 227-229

25 Singh S, Singh R, Wahajuddin, Jain G. Development and validation of a rapid, sensitive liquid chromatography-tandem mass spectrometry method using electrospray ionization for quantitation of centchroman in rat plasma and its application to preclinical pharmacokinetic study. J Chromatogr B 2008; 876: 1-7

26 Wahajuddin, Singh S, Jain G. Determination of lumefantrine in rat plasma by liquid-liquid extraction using LC-MS/MS with electrospray ionization: assay development, validation and application to a pharmacokinetic study. J Chromatogr B 2009; 877: 1133-1139
27 Yang W, Feng C, Kong DZ, Shi XW, Cui Y, Liu M, Wang Q Wang YL, Zhang $L T$. Simultaneous and sensitive determination of xanthotoxin, psoralen, isoimpinellin and bergapten in rat plasma by liquid chromatography-electrospray ionization mass spectrometry. J Chromatogr B 2010; 878: $575-582$

28 Dams R, Huestis MA, Lambert WE, Murphy CM. Matrix effect in bioanalysis of illicit drugs with LC-MS/MS: influence of ionization type, sample preparation, and biofluid. J Am Soc Mass Spectrom 2003; 14 : 1290-1294

29 Taylor P. Matrix effects: the Achilles heel of quantitative high-performance liquid chromatography-electrospray-tandem mass spectrometry. Clin Biochem 2005; 38: 328-334 